\title{
Angiopoietin 2 as a therapeutic target in hepatocellular carcinoma treatment: current perspectives
}

This article was published in the following Dove Press journal:

OncoTargets and Therapy

20 October 2014

Number of times this article has been viewed

\section{Manojkumar Bupathi' \\ Ahmed Kaseb ${ }^{2}$ \\ Filip Janku'}

'Department of Investigational Cancer Therapeutics (Phase I Clinical Trials Program), ${ }^{2}$ Gastrointestinal Medical Oncology, The University of Texas MD Anderson Cancer Center, Houston, TX, USA
Correspondence: Filip Janku

Department of Investigational Cancer

Therapeutics (Phase I Clinical Trials

Program), The University of Texas MD

Anderson Cancer Center,

I5I 5 Holcombe Blvd, Unit 455,

Houston, TX 77030, USA

$\mathrm{Tel}+\mathrm{I} 7135632632$

Fax +I $7 / 3745805$

Email fjanku@mdanderson.org

\begin{abstract}
Hepatocellular carcinoma (HCC) is a hypervascular malignancy and is the third leading cause of death worldwide. The disease has multiple predisposing risk factors and limited treatment options. Although the precise mechanism(s) underlying HCC is unknown, several pathways have been implicated in its development. HCC is a vascular tumor and angiogenesis is believed to play an important role in its progression. Hypoxia is believed to increase the expression of vascular endothelial growth factor (VEGF) through the expression of the hypoxia-inducible factor-1 $\alpha$. VEGF and angiopoietin 2 (Ang2) are expressed on cancer cells, whereas angiopoietin 1 (Ang1) occurs predominantly in support cells of large blood cells as well as stromal, endothelial, and tumor cells. Ang2 is concomitantly an agonist and antagonist of angiopoietin 1 and is expressed during vascular remodeling. This prevents vascular stability and allows VEGF to stimulate endothelial cells. Ang2 is expressed along with growing blood vessels, destabilizing the vascular integrity. Inhibiting Ang2 can, on the other hand, promote vesicular stability and decrease angiogenesis. We discuss in this review angiopoietin as a therapeutic target alone or in combination with other therapies.
\end{abstract}

Keywords: hepatocellular carcinoma, AKT, angiopoietin 1/2, mTOR, PI3K, sorafenib, VEGF

\section{Introduction}

Hepatocellular carcinoma (HCC) is a hypervascular malignancy arising from liver parenchyma and is the third leading cause of death worldwide. ${ }^{1,2}$ This disease has underlying causative risk factors including hepatitis $\mathrm{B}$ and $\mathrm{C}$, aflatoxins, and alcoholic and nonalcoholic steatohepatitis. ${ }^{3,4}$ Surgery is the only curative treatment in patients with limited disease and the therapeutic options available for patients with advanced or metastatic HCC are limited. Sorafenib is the only systemic therapy approved by the US Food and Drug Administration for advanced HCC. ${ }^{5}$

Although the principal pathogenic mechanisms of HCC are complex, pathways such as vascular endothelial growth factor (VEGF)-mediated angiogenesis, Wnt, $\mathrm{PI} 3 \mathrm{~K} / \mathrm{AKT} / \mathrm{mTOR}$, AMP-activated protein kinase, and C-MET are known to play a major role in HCC. Consequently, numerous molecular abnormalities can produce numerous potential therapeutic targets. ${ }^{5,6} \mathrm{HCC}$ is a vascular tumor and tumor angiogenesis is important in the invasion, metastasis, and progression of the disease. ${ }^{7}$ Angiogenesis in HCC is believed to result from an imbalance between angiogenic and antiangiogenic factors. ${ }^{8} \mathrm{CD} 34$, a sensitive angiogenesis-related endothelial marker, is not typically found in healthy liver and is rarely identified in cirrhotic liver; however, its levels are increased in HCC, implicating angiogenesis as a causative factor. ${ }^{9}$ 


\section{Angiopoietin and its role in HCC}

In HCC, hypoxia is believed to increase the expression of VEGF through the expression of hypoxia-inducible factor- $1 \alpha \cdot{ }^{10}$ VEGF and its receptor control the early steps triggering the angiogenic cascade. VEGF is the best known angiogenic factor involved in angiogenesis, promoting endothelial cell migration/proliferation. ${ }^{10}$ It is also known as a vascular permeability factor that stimulates endothelial cell proliferation specifically through tyrosine kinase receptors. ${ }^{11}$ Sorafenib targets the VEGF-mediated and Raf-MAPK/ERK pathways. ${ }^{6}$

Angiopoietin 1 (Ang1) and angiopoietin 2 (Ang2) are ligands for the tyrosine kinase receptor Tie2 and are widely expressed in many embryonic tissues. Transcription of Ang2 is however restricted in adult tissues. ${ }^{12}$ VEGF and Ang2 are expressed on cancer cells, whereas Ang1 is found predominantly in the support cells of large blood cells as well as stromal, endothelial, and tumor cells. Ang2 is a partial agonist and antagonist of Ang1 and is expressed during vascular remodeling, thus preventing vascular stability. Consequently, VEGF is able to stimulate endothelial cells. ${ }^{13}$ In normal physiology, there is a homeostatic balance between Ang1 and Ang2. ${ }^{14}$ Ang1 promotes recruitment of pericytes and smooth muscle cells, stabilizing vascular networks by binding to Tie2. ${ }^{10,15}$ Ang1 binds to Tie2, which is constitutively expressed on stromal cells such as fibroblasts and vascular supporting cells, and by non-vascular supporting cells. ${ }^{16}$ Ang2 is also produced and stored within the Weibel-Palade storage granules of endothelial cells. ${ }^{14} \mathrm{~A}$ study by Reiss et a ${ }^{17}$ showed that overexpression of Ang2 decreases phosphorylation of Tie2. Moreover, Ang2 competitively inhibits Ang1-induced activation of the Tie 2 receptor, thus relaxing the interactions among endothelial and perivascular support cells and extracellular matrix, abrogating vascular integrity. ${ }^{10}$ Further activation of the Tie 2 receptor leads to signal transduction, which promotes endothelial cell survival, proliferation, and motility, all of which promote angiogenesis. ${ }^{18}$ In contrast, there is also some evidence that Ang2 delivers proangiogenic activity through binding the Tie 2 receptor, specifically in monocytes. ${ }^{19,20}$ Some Tie2-expressing monocytes related to tumor blood vessels produce angiogenic activity that can be inhibited by blocking Tie2 or Ang2 (Figure 1). ${ }^{19,20}$

Angiogenic factors and the Tie2 pathway regulate the relationship between blood vessel stabilization and remodeling. ${ }^{10}$ In the absence of VEGF, binding of Ang2 to Tie2 destabilizes the cell matrix and may induce endothelial cell apoptosis and vascular regression. In the presence of

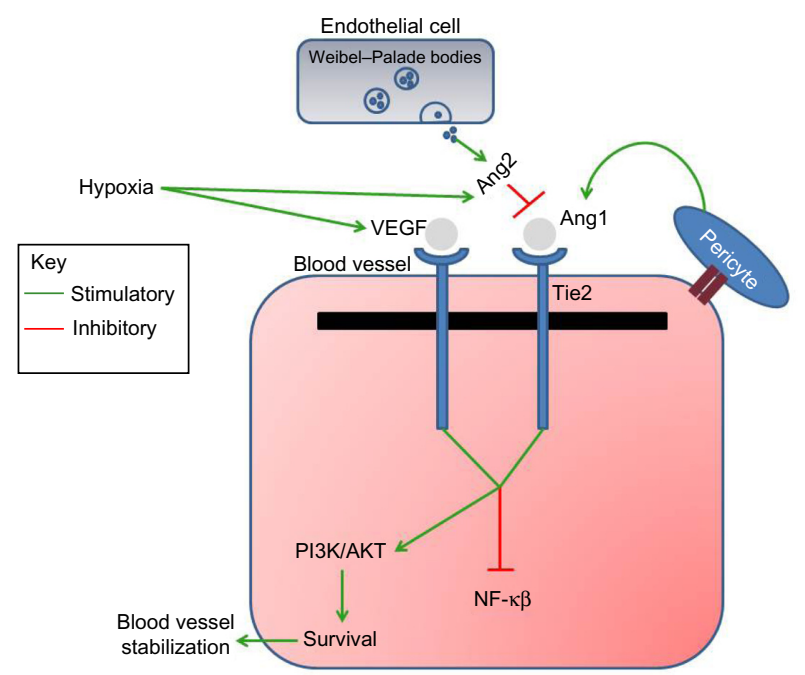

Figure I Schematic showing the signal transduction with Tie2 and Angl/2. Notes: Proposed model showing green arrows (stimulatory effect) and red arrows (inhibitory effect). Tie2 is expressed on the blood vessel and, when Ang I bind causes a stimulatory effect, thus downstream signal transduction. Ang2 has an inhibitory effect on Angl.

Abbreviations: Ang I, angiopoietin I; Ang2, angiopoietin 2; VEGF, vascular endothelial growth factor.

VEGF, binding of Ang2 to Tie2 causes endothelial cell migration and proliferation, and a permissive angiogenic signal.

Lin et $\mathrm{a}^{21}$ have shown that when the functionality of Tie 2 function is disrupted in transgenic mice, embryonal death results from defects in vascular development, characterization, and the morphogenesis of microvessels. The authors also demonstrated that by blocking Tie 2 activation via a soluble Tie2 receptor, they were able to inhibit tumor angiogenesis and growth. A study by Tanaka et $\mathrm{al}^{12}$ showed that Tie2 and Ang1 knockout mice have immature vascularization patterns and abatement of support cells. In this study, they also found that Ang2 competitively inhibits Ang1's ability to bind to Tie2, showing that Ang2 is an antagonist to Ang1. These authors demonstrated that when Ang2 is overexpressed in transgenic mice, the vascular system fails to mature, which is similar to what was seen in Tie 2 and Ang1 knockout mice. On the basis of these findings, they concluded that Ang1 activates Tie2 to promote interaction with perivascular support cells such as pericytes. In contrast, Ang2 reduces matrix contacts and interactions with support cells, and may very well be essential for vascular remodeling in HCC. The same authors later published another study where they looked at Tie2 expression by gene transfer in murine HCC tumors and evaluated tumor growth. In this study, immunohistochemistry staining showed evidence of Tie 2 in 31 (80\%) of 39 HCC samples (24/27, 89\% moderately differentiated tumors; $4 / 6,67 \%$ poorly differentiated tumors; and 1/6, 17\% well differentiated tumors). Tanaka 
et $\mathrm{al}^{22}$ showed that the Tie2 signaling pathway is involved in $\mathrm{HCC}$ and is a potential therapeutic target.

\section{Angiopoietin as a prognostic factor}

A study by Scholz et $\mathrm{al}^{23}$ indicated that Ang2 levels were elevated in patients with HCC and cirrhosis. Furthermore, increased levels of Ang2 are associated with advanced pathological features and worse overall survival (OS) as shown by a study from Llovet et al. ${ }^{24}$ In this Phase III study, patients with advanced or metastatic HCC were randomized to sorafenib or placebo and one of the correlative objectives was to explore the plasma biomarkers to predict prognosis. In both cohorts, baseline angiopoietin and VEGF concentrations independently predicted survival. ${ }^{6,24}$ Ang2 is a ligand of the Tie2 tyrosine-kinase receptor, which was reported to promote angiogenesis and tumor growth. ${ }^{25,26}$ Recent studies showed elevated plasma expression of Ang2, ${ }^{23}$ and upregulation of Ang2 messenger RNA. ${ }^{10,13}$

\section{Angiopoietin as a therapeutic target}

As mentioned, Ang2 is expressed concomitantly with growing blood vessels by destabilizing the blood vessels; inhibition of Ang2 can promote microvascular stability and decrease angiogenesis. ${ }^{27}$ A study by Hashizume et $\mathrm{al}^{27}$ looked at selective inhibition of Ang2 by the peptide fusion protein L1-7(N), which reduced vascular sprouts by $46 \%$ and tumor growth by $62 \%$ over a period of 26 days in a nude mouse model. When L1-7(N) was combined with VEGF antibody, the number of sprouts (mechanism of blood vessel growth) was decreased by $82 \%$, tumor vascularity by $67 \%$, and tumor growth by 91\% compared to controls. ${ }^{27}$ Further, another drug, AMG 386 , is a peptide-Fc fusion protein, which inhibits Ang1 and Ang2 binding with Tie2. ${ }^{18}$ A Phase I study by Herbst et al ${ }^{18}$ assessed the activity of AMG 386 in patients with advanced solid tumors. Although the maximum tolerated dose was not reached, one patient with refractory ovarian cancer had a partial response with a $32.5 \%$ reduction in target lesions, and four patients with diverse solid tumors had stable disease for at least 16 weeks. Additionally, none of the patients had a diagnosis of $\mathrm{HCC}$.

\section{Harnessing resistance to antiangiogenic therapy}

Progression of disease in HCC can occur due to resistance to therapy, which can be driven by the activity of alternative signaling pathways or neovascularization. HCC is often associated with a complex activation of multiple cellular pathways involved in tumorigenesis, which is impossible to control with single-agent blockade.

In the past, antiangiogenic therapy was believed to cause tumor regression by targeting tumor-associated vasculature. In actuality, this has not been the case and clinically most antiangiogenic therapies promote stable disease at best since they target the formation of new, immature blood vessels. In addition, treatment with antiangiogenic agents alone can increase circulating levels of cytokines, including angiopoietin 1, which might abrogate treatment effects. Ellis and Hicklin ${ }^{28}$ theorize that many cytokines, including Ang1, stromal cell-derived factor 1, and basic fibroblast growth factor (bFGF), are important in tumor-cell invasion and metastasis. Anti-VEGF therapy results in hypoxic destruction of blood vessels leading to increased proangiogenic and promigratory factors; thus, treatment with anti-VEGF therapy alone may not be sufficient to control tumor growth. ${ }^{28}$

Sorafenib, an oral multikinase and VEGF receptor tyrosine kinase inhibitor, has activity in subsets of patients with HCC and is the only US Food and Drug Administrationapproved drug currently used for advanced/metastatic HCC. In a pivotal Phase III study, patients with advanced HCC treated with sorafenib demonstrated prolonged OS compared to placebo (10.7 months versus 7.9 months, hazard ratio [HR] $0.78, P=0.0041) .{ }^{24}$ Unfortunately, all patients, irrespective of initial response, ultimately develop resistance, plausibly due to activation of diverse alternative signaling pathways. For example, elevated expression levels of Ang2 were shown to be associated with resistance to antiangiogenesis and poor OS in HCC. ${ }^{6,24}$ The retrospective biomarker analysis of 491 advanced HCC patients treated in the randomized Phase III trial of sorafenib versus placebo, which included ten plasma biomarkers, such as VEGF, soluble VEGFR-2, soluble VEGFR-3, soluble c-KIT, soluble Ras, Ang2, bFGF, epidermal growth factor, insulin-like growth factor-2, and hepatocyte growth factor, showed in multivariate analysis that increased levels of soluble VEGF and Ang2 were independent prognostic factors for shorter median OS. ${ }^{24}$ In all patients, irrespective of treatment arm, low baseline Ang2 levels demonstrated a longer median OS compared to patients with high baseline Ang2 levels (14.1 months versus 6.3 months; $P<0.001)$. Similarly, patients with low levels of VEGF had a longer median OS of 10.6 months compared to 6.2 months in patients with high levels of VEGF $(P<0.001)$. Both high levels of Ang2 and VEGF were confirmed as independent negative prognostic factors in a multivariate analysis 
(HR 1.58, $P=0.001$ and HR 1.48, $P=0.015$, respectively). Furthermore, increased Ang2 levels while on study were associated with shorter time-to-progression and OS in the sorafenib ( $P=0.0002, P<0.0001$, respectively) and placebo groups ( $P<0.0001, P<0.0001$, respectively), reflecting that Ang2 is a marker of poor prognosis irrespective of therapeutic intervention.

This is not surprising since high levels of plasma Ang2 in combination with high VEGFA levels can also be associated with poor survival in other cancers such as breast and non-small cell lung cancer, providing a rationale for Ang2 and anti-VEGFA targeted therapy. ${ }^{24,29}$ Nevertheless, none of the markers, including Ang2, were able to predict response to sorafenib.

Because of limitations of current therapeutic strategies targeting angiogenesis in HCC, several novel compounds are under development (Table 1). For example, a Phase I dose-escalation study in patients with advanced cancers with a novel human monoclonal antibody, AMG 780, which disrupts interaction between Ang1/2 and Tie2 is underway. Another study using AMG 386 and abiraterone is being conducted in prostate cancer; however, no $\mathrm{HCC}$ recruitment is planned as of now.

An abstract of a study of Kelley et $\mathrm{al}^{30}$ showed waning AFP levels $(2 / 15,13 \%)$ and prolonged stable disease $(6 / 15,40 \%)$ in 15 patients with advanced HCC treated with a monoclonal human antibody against Ang2 called nesvacumab. In a Phase II study done by Faivre et al, ${ }^{31}$ transforming growth factor-beta receptor I kinase inhibitor, LY2157299, had promising effects in patients with HCC. In this study, 109 sorafenib pretreated or naïve patients (Child-Pugh score $\mathrm{A} / \mathrm{B}[97 \% / 3 \%]$, and AFP $\geq 1.5$ upper limit of normal) were enrolled. A median time-to-progression for sorafenib-naïve patients was 18.3 weeks and was 12 weeks for the overall population; however, there was no formal statistical evaluation done. In addition, a maximum decrease of AFP by $\geq 20 \mathrm{ng} / \mathrm{mL}$ was seen in $24 \%$ of patients and those with decreased levels of AFP had a median OS of 93.1 weeks compared to 29.6 weeks in patients without a decrease in $\operatorname{AFP}(P=0.0006)$.

\section{Challenges with antiangiogenic agents}

While the use of antiangiogenic therapy is appealing, there are a few challenges which can potentially arise. Antiangiogenic agents cause vascular trimming and intratumoral hypoxia, and thus have a limited efficacy as single agents in many malignancies and can lead to intrinsic and acquired resistance. ${ }^{32}$ Intrinsic resistance is defined as tumor growth despite treatment with an antiangiogenic agent and an example of acquired resistance is resistance to therapy due to resistance clones with an alternative signaling mechanism. For instance, patients can be resistant to anti-VEGF therapy or respond minimally like those with pancreatic cancer. ${ }^{33}$ Antiangiogenic factors, specifically anti-VEGF, can cause endothelial dysfunction in normal-health tissues which can be aggravated when these drugs are combined with other targeted agents. ${ }^{34}$ Furthermore, Van der Veldt et $\mathrm{al}^{35}$ showed that antiangiogenic agents can decrease the delivery of cytotoxic drugs to tumors, thus decreasing the therapeutic benefit. These authors evaluated patients with non-small cell lung cancer treated with bevacizumab. In these patients, VEGF inhibition decreased the penetration of cytotoxic agents. Since Ang2 is also an antiangiogenic target, similar effects can possibly be seen.

\section{Future directions}

Myriad signaling pathways are involved in the pathogenesis of HCC and their therapeutic targeting is currently being

Table I Ang2/Tie2 targeting agents

\begin{tabular}{|c|c|c|c|c|c|}
\hline Compound & Class & Target & Status & Cancer type & NCT numbers \\
\hline AMG $386^{16}$ & Peptide & Angl, Ang2 & Phase III & Ovarian & 01493505 \\
\hline MEDI36I $17^{16}$ & Monoclonal antibody & Ang2 & Phase I/lb & All solid tumors & 01248949 \\
\hline AMG $780^{16}$ & Monoclonal antibody & Ang2/Tie2 & Completed & All solid tumors & 01137552 \\
\hline REGN910 16 & Monoclonal antibody & Ang2 & Phase I & All solid tumors & 01271972 \\
\hline Arry-614 $4^{16}$ & Tyrosine kinase inhibitor & Tie2 & Phase I & Myelodysplastic syndromes & 01496495 \\
\hline CVX-060 16 & Monoclonal CovX body & Ang2 & Completed & Renal cell carcinoma & 00982657 \\
\hline Axitinib $^{14}$ & Tyrosine kinase inhibitor & VEGF & Phase lb & All solid tumors & 01999972 \\
\hline Regorafenib ${ }^{16}$ & Tyrosine kinase inhibitor & Tie2, VEGFR, FGFR & Phase I & All solid tumors & 02095054 \\
\hline $\mathrm{XLI} 84^{14}$ & Tyrosine kinase inhibitor & Tie2, VEFGR & Phase I & $\mathrm{HCC}$ & 01908426 \\
\hline GSKI $363089^{14}$ & Tyrosine kinase inhibitor & VEGF, Tie2 & Phase I/II & $\mathrm{HCC}$ & 00920192 \\
\hline CEP-I $|98|^{14}$ & Tyrosine kinase inhibitor & VEGF, Tie2 & Phase I & Advanced cancers & 00875264 \\
\hline TAvi6 ${ }^{14}$ & Tetravalent antibody & Ang2 & Preclinical & None & None \\
\hline
\end{tabular}

Abbreviations: AngI, angiopoietin I; Ang2, angiopoietin 2; HCC, hepatocellular carcinoma; VEGF, vascular endothelial growth factor; R, receptor; FGF, fibroblast growth factor. 
evaluated in more than 35 clinical studies. ${ }^{36}$ Angiogenesis remains one of the most promising therapeutic targets since Ang2 along with VEGF work together to promote tumor progression in HCC and other malignancies. Therefore, novel therapies and combinations targeting Ang2 and/or VEGF/ VEGFR have the potential to lead to therapeutic advances. VEGF and Ang2 pathways can result in downstream activation of the PI3K/AKT/mTOR pathway, NF- $\kappa \beta$, and others, which provides a further rationale for developing novel combinations with the potential of overcoming therapeutic resistance.

Antiangiogenic therapies such as Ang2 inhibitors can also be tested in combination with conventional chemotherapy, albeit with a caveat that chemotherapy has traditionally had a limited role in the treatment of HCC in the setting of advanced cirrhosis. Alternatively, combinations of antiangiogenics including Ang2 inhibitors with locoregional approaches, such as transarterial chemoembolization, hepatic arterial infusion, or yttrium-90, can further improve treatment outcomes in patients with unresectable HCC.

It is widely known that cancer in general is a disease driven by diverse genetic abnormalities. Novel technologies, such as next-generation sequencing, can identify mutations within the genome, subsequently leading to a better understanding of tumorigenesis and development of rationally designed targeted therapies. The etiology of HCC is varied among patients; similarly, the molecular prolife of all patients can be different. A study analyzed tumor samples using next-generation sequencing and outcomes for patients with advanced/metastatic HCC. ${ }^{37}$ The authors evaluated 14 patients, most of whom had hepatitis B/C-related HCC; 12/14 (86\%) patients were treated with targeted agents. Despite the small number of patients, gain of function mutations, loss of function mutations, copy number variations, and gene rearrangements were identified across a number of different pathways, for example, Wnt and PI3K/mTOR. The identification of numerous mutations across this small group of patients only hints at the complex biology that drives the development of HCC, pointing to the potential need to do molecular testing of all patients.

While various combination therapies seem attractive, many challenges will need to be addressed in the future. For example, which combination therapy is best suited for which patient? Would a similar class of drug used in a different combination be better? Furthermore, as cancer care is advancing, genomic and personalized medicine is entering the foreground as the most appropriate diagnostic strategy. Other hurdles to overcome will be how to choose a therapy for a patient with more than one activation mutation. Should such a patient be treated with a single agent or combination therapy? How long will it take for a patient to develop resistance to a given treatment regimen? These important questions will need to be addressed in upcoming clinical trials.

\section{Acknowledgments}

We thank Ms Joann Aaron for scientific review and editing of this article. The authors wish to acknowledge the Jeanne F Shelby Scholarship Fund for Investigational Cancer Therapeutics Fellowship.

\section{Disclosure}

The authors report no conflicts of interest in this work.

\section{References}

1. Parkin DM, Bray F, Ferlay J, Pisani P. Estimating the world cancer burden: Globocan 2000. Int J Cancer. 2001;94(2):153-156.

2. El-Serag HB. Hepatocellular carcinoma. N Engl J Med. 2011;365(12): $1118-1127$.

3. Matsubara T, Kanto T, Kuroda S, et al. TIE2-expressing monocytes as a diagnostic marker for hepatocellular carcinoma correlates with angiogenesis. Hepatology. 2013;57(4):1416-1425.

4. Buitrago-Molina LE, Vogel A. mTor as a potential target for the prevention and treatment of hepatocellular carcinoma. Current Cancer Drug Targets. 2012;12(9):1045-1061.

5. Llovet JM, Ricci S, Mazzaferro V, et al. Sorafenib in advanced hepatocellular carcinoma. $N$ Engl J Med. 2008;359(4):378-390.

6. Kaseb AO, Garrett-Mayer E, Morris JS, et al. Efficacy of bevacizumab plus erlotinib for advanced hepatocellular carcinoma and predictors of outcome: final results of a phase II trial. Oncology. 2012;82(2): $67-74$.

7. Ji Y, Wang Z, Li Z, Li K, Le X, Zhang T. Angiotensin II induces angiogenic factors production partly via AT1/JAK2/STAT3/SOCS3 signaling pathway in MHCC97H cells. Cell Physiol Biochem. 2012;29(5-6): 863-874.

8. Sharma BK, Srinivasan R, Kapil S, et al. Serum levels of angiogenic and anti-angiogenic factors: their prognostic relevance in locally advanced hepatocellular carcinoma. Mol Cell Biochem. 2013;383(1-2): 103-112.

9. Chen L, Yang Z, Wang G, Wang C. Expression of angiopoietin-2 gene and its receptor Tie2 in hepatocellular carcinoma. J Tongji Med Univ. 2001;21(3):228-230, 235

10. Torimura T, Ueno T, Kin M, et al. Overexpression of angiopoietin-1 and angiopoietin-2 in hepatocellular carcinoma. J Hepatol. 2004;40(5): 799-807.

11. Ferrara N, Davis-Smyth T. The biology of vascular endothelial growth factor. Endocr Rev. 1997;18(1):4-25.

12. Tanaka S, Mori M, Sakamoto Y, Makuuchi M, Sugimachi K, Wands JR. Biologic significance of angiopoietin-2 expression in human hepatocellular carcinoma. J Clin Invest. 1999;103(3): 341-345.

13. Moon WS, Rhyu KH, Kang MJ, et al. Overexpression of VEGF and angiopoietin 2: a key to high vascularity of hepatocellular carcinoma? Mod Pathol. 2003;16(6):552-557.

14. Gerald D, Chintharlapalli S, Augustin HG, Benjamin LE. Angiopoietin-2: an attractive target for improved antiangiogenic tumor therapy. Cancer Res. 2013;73(6):1649-1657. 
15. Papapetropoulos A, García-Cardeña G, Dengler TJ, Maisonpierre PC, Yancopoulos GD, Sessa WC. Direct actions of angiopoietin-1 on human endothelium: evidence for network stabilization, cell survival, and interaction with other angiogenic growth factors. Lab Invest. 1999;79(2):213-223.

16. Cascone T, Heymach JV. Targeting the angiopoietin/Tie2 pathway: cutting tumor vessels with a double-edged sword? J Clin Oncol. 2012;30(4):441-444.

17. Reiss Y, Droste J, Heil M, et al. Angiopoietin-2 impairs revascularization after limb ischemia. Circ Res. 2007;101(1):88-96.

18. Herbst RS, Hong D, Chap L, et al. Safety, pharmacokinetics, and antitumor activity of AMG 386, a selective angiopoietin inhibitor, in adult patients with advanced solid tumors. J Clin Oncol. 2009;27(21): 3557-3565.

19. Mazzieri R, Pucci F, Moi D, et al. Targeting the ANG2/TIE2 axis inhibits tumor growth and metastasis by impairing angiogenesis and disabling rebounds of proangiogenic myeloid cells. Cancer Cell. 2011;19(4):512-526.

20. Venneri MA, De Palma M, Ponzoni M, et al. Identification of proangiogenic TIE2-expressing monocytes (TEMs) in human peripheral blood and cancer. Blood. 2007;109(12):5276-5285.

21. Lin P, Buxton JA, Acheson A, et al. Antiangiogenic gene therapy targeting the endothelium-specific receptor tyrosine kinase Tie2. Proc Natl Acad Sci USA. 1998;95(15):8829-8834.

22. Tanaka S, Sugimachi K, Yamashita Yi Y, et al. Tie2 vascular endothelial receptor expression and function in hepatocellular carcinoma. Hepatology. 2002;35(4):861-867.

23. Scholz A, Rehm VA, Rieke S, et al. Angiopoietin-2 serum levels are elevated in patients with liver cirrhosis and hepatocellular carcinoma. Am J Gastroenterol. 2007;102(11):2471-2481.

24. Llovet JM, Peña CE, Lathia CD, et al. Plasma biomarkers as predictors of outcome in patients with advanced hepatocellular carcinoma. Clin Cancer Res. 2012;18(8):2290-2300.

25. Maisonpierre PC, Suri C, Jones PF, et al. Angiopoietin-2, a natural antagonist for Tie2 that disrupts in vivo angiogenesis. Science. 1997; 277(5322):55-60.

26. Yu Q, Stamenkovic I. Angiopoietin-2 is implicated in the regulation of tumor angiogenesis. Am J Pathol. 2001;158(2):563-570.
27. Hashizume H, Falcón BL, Kuroda T, et al. Complementary actions of inhibitors of angiopoietin-2 and VEGF on tumor angiogenesis and growth. Cancer Res. 2010;70(6):2213-2223.

28. Ellis LM, Hicklin DJ. Resistance to Targeted Therapies: Refining Anticancer Therapy in the Era of Molecular Oncology. Clin Cancer Res. 2009;15(24):7471-7478.

29. Imanishi Y, Hu B, Jarzynka MJ, et al. Angiopoietin-2 stimulates breast cancer metastasis through the alpha(5)beta(1) integrin-mediated pathway. Cancer Res. 2007;67(9):4254-4263.

30. Kelley RK, Siu LL, Van Loon K, et al. Phase 1 first-in-human (FIH) study of nesvacumab (REGN910) a fully human and selective angiopoietin-2 (Ang2) monoclonal antibody (MAb): Results from hepatocellular carcinoma (HCC) cohort. J Clin Oncol. 2014; 32(Suppl; abstr 2540):5s.

31. Faivre SJ, Santoro A, Kelley RK, et al. A phase 2 study of a novel transforming growth factor-beta (TGF- $\beta 1$ ) receptor I kinase inhibitor, LY2157299 monohydrate (LY), in patients with advanced hepatocellular carcinoma (HCC). J Clin Oncol. 2014;32(Suppl 3; abstr LBA173).

32. Moserle L, Jiménez-Valerio G, Casanovas O. Antiangiogenic therapies: going beyond their limits. Cancer Discov. 2014;4(1):31-41.

33. Kindler HL. Pancreatic cancer: an update. Curr Oncol Rep. 2007;9(3): 170-176.

34. Hurwitz H, Fehrenbacher L, Novotny W, et al. Bevacizumab plus irinotecan, fluorouracil, and leucovorin for metastatic colorectal cancer. N Engl J Med. 2004;350(23):2335-2342.

35. Van der Veldt AA, Lubberink M, Bahce I, et al. Rapid decrease in delivery of chemotherapy to tumors after anti-VEGF therapy: implications for scheduling of anti-angiogenic drugs. Cancer Cell. 2012;21(1):82-91.

36. Galuppo R, Ramaiah D, Ponte OM, Gedaly R. Molecular therapies in hepatocellular carcinoma: what can we target? Dig Dis Sci. 2014;59(8):1688-1697.

37. Janku F, Kaseb AO, Tsimberidou AM, Wolff RA, Kurzrock R. Identification of novel therapeutic targets in the PI3K/AKT/mTOR pathway in hepatocellular carcinoma using targeted next generation sequencing. Oncotarget. 2014;5(10):3012-3022.
OncoTargets and Therapy

\section{Publish your work in this journal}

OncoTargets and Therapy is an international, peer-reviewed, open access journal focusing on the pathological basis of all cancers, potential targets for therapy and treatment protocols employed to improve the management of cancer patients. The journal also focuses on the impact of management programs and new therapeutic agents and protocols on

\section{Dovepress}

patient perspectives such as quality of life, adherence and satisfaction The manuscript management system is completely online and includes a very quick and fair peer-review system, which is all easy to use. Visit http://www.dovepress.com/testimonials.php to read real quotes from published authors. 